\title{
The Approximate Solution of Heat Radiation in A Semi-infinite Solid by using Shifted Chebyshev Polynomials of the First kind
}

\author{
1 Wafaa faeik keidan \\ ${ }^{1}$ Diyala University, Collage of Education Almqdad, Department of Mathematics.
}

Correspondence Author: Wafaa faeik keidan, Diyala University, collage of Education Almqdad, Department of Mathematics.

E-mail: Assola_fa@yahoo.com

Received date: 23 February 2018, Accepted date: 1 April 2018, Online date: 15 April 2018

Copyright: ( $) 2018$ Wafaa faeik keidan. This is an open-access article distributed under the terms of the Creative Commons Attribution License, which permits unrestricted use, distribution, and reproduction in any medium, provided the original author and source are credited.

\begin{abstract}
In this paper, we use Shifted Chebyshev polynomials of the first kind method to solve on linear Volterra weakly singular integral equations (NLVWSIE's) of the second kind that described heat radiation. This techniques transform the Non linear Volterra weakly singular integral equations to a system of nonlinear algebraic equations. Example is presented to illustrate the efficiency and accuracy of this method.
\end{abstract}

Keywords: Nonlinear Volterra integral equations, Weakly singular kernel, Heat Radiation, Shifted Chebyshev polynomials.

\section{INTRODUCTION}

Any functional equation in which the unknown function appears under the sign of integration is called an integral equation. Integral equations arise in a great many branches of science; for example, in potential theory, acoustics, elasticity, fluid mechanics, radiative transfer, theory of population, etc (Abdul, 2011).

Abel's equation is one of the integral equation derived directly from a concrete problem of mechanics or physics (without passing through a differential equation). Historically, Abel's problem is the first one that led to the study of integral equations. The generalized Abel's integral equations on a finite segment appeared in the paper of Zeilon (N. Zeilon, 1924) for the first time.

A comprehensive reference on Abel-type equations, including an extensive list of applications, can be found in R. (Gorenflo, Vessella, 1991) and ( $\mathrm{R}$ Gorenflo, Vessella, 1991)

Also, many researchers have been studied heat transfer application say.

In 1985, Franco and Mckee, (Franco, Mckee, 1985) used a family of high order product integration methods to solve integral equation of Light hill. In 2006, Tao and Yong, (L.Tao and H.Yong, 2006) used Extrapolation method for solving NLVWSIEs of the second kind which arises in a heat transfer problem. In 2010, Abdul - Majid Wazwaz and Mona S.Mehanna, (Abdul, Mona, Mehanna, 2010) used the combined Laplace - Adomian method for Handling SIE of heat transfer. In 2010, Rebelo and Diogo, (M. Rebelo, Diogo, 2010) used a hybrid collocation method for solving nonlinear Volterra integral equation with weakly singular kernel. The rest of this paper is organized as follows. In the section two Chebyshev polynomials of the first kind. In the section three modes of heat transfer. In the section four the proposed method for solving nonlinear Volterra weakly singular integral equations. Example is presented in section five. Finally, a brief Conclusion is stated in last section.

2.Chebyshev polynomials of the first kind $T_{n}(x)$, (Mason, Handscomb, 2003):

2.1Definition

Chebyshev polynomialof degree $n \geq 0$ is defined as

$$
T_{n}(x)=\cos (n \arccos x), x \in[-1,1]
$$

Or, in a more instructive form $, T_{n}(x)=\cos n \theta, x=\cos \theta, \theta \in[0, \pi]$

2.2 Properties of the Chebyshev polynomials $T_{n}(x)$ :

1. The Chebyshev polynomials of the first kind $T_{n}(x), n=0,1, \ldots$

are a set of orthogonal polynomials over the interval $[-1,1]$ with respect to the weight function $w(x)=\left(1-x^{2}\right)^{-1 / 2}$, that is :

$\int_{-1}^{1} \frac{T_{n}(x) T_{m}(x)}{\sqrt{1-x^{2}}}=\left\{\begin{array}{lr}0 & n \neq m \\ \frac{\pi}{2} & n=m \neq 0 \\ \pi & n=m=0\end{array}\right.$

2. $T_{n}(x)$ has $\mathrm{n}$ distinct real roots $x_{i}$ on the interval [-1,1], these roots are defined by:

$x_{i}=\cos \left(\frac{(2 i+1)}{2 N}\right), i=0,1,2, \ldots, N-1$

are called Chebyshev nodes. $T_{n}(x)$ assumes its absolute extrema at

$x_{j}=\cos \left(\frac{j \pi}{N}\right)$ for $j=0,1,2, \ldots, N$

3.Recursive relation of chebyshev polynomials $T_{0}(x)=1, T_{1}(x)=x, T_{n+1}(x)=2 x T_{n}(x)-T_{n-1}(x), n \geq 1$

4.Apolynomial of degree $\mathrm{N}$ in Chebyshev form is a polynomial $y(x)=\sum_{n=0}^{N} c_{n} T_{n}(x)$

Where $T_{n}$ is the $n^{\text {th }}$ Chebyshev form 
2.3 Shifted to any interval:

The transformation $\bar{x}=\frac{1}{2}[(b-a) x+(a+b)]$

$T_{n}^{*}(x)=T_{n}(2 x-1), \quad 0 \leq x \leq 1$

Transforms the nodes $x_{i}$ in $[-1,1]$ in to the corresponding nodes $\bar{x}_{i}$ in $[a, b]$.

\section{Heat Transfer(Geankoplis, Christie, 2003):}

Heat transfer is a discipline of thermal engineering that concerns the generation, use, conversion, and exchange of thermal energy (heat) between physical systems. Heat transfer is classified into various mechanisms, such as thermal conduction, thermal convection, thermal radiation, and transfer of energy by phase changes. Engineers also consider the transfer of mass of differing chemical species, either cold or hot, to achieve heat transfer. While these mechanisms have distinct characteristics, they often occur simultaneously in the same system.

Heat conduction, also called diffusion, is the direct microscopic exchange of kinetic energy of particles through the boundary between two systems. When an object is at a different temperature from another body or its surroundings, heat flows so that the body and the surroundings reach the same temperature, at which point they are in thermal equilibrium. Such spontaneous heat transfer always occurs from a region of high temperature to another region of lower temperature, as described in the second law of thermodynamics.

Heat convection occurs when bulk flow of a fluid (gas or liquid) carries heat along with the flow of matter in the fluid. The flow of fluid may be forced by external processes, or sometimes (in gravitational fields) by buoyancy forces caused when thermal energy expands the fluid (for example in a fire plume), thus influencing its own transfer. The latter process is often called "natural convection". All convective processes also move heat partly by diffusion, as well. Another form of convection is forced convection. In this case the fluid is forced to flow by use of a pump, fan or other mechanical means.

Thermal radiation occurs through a vacuum or any transparent medium (solid or fluid). It is the transfer of energy by means of photons in electromagnetic waves governed by the same laws.

4.The Approximate Solution of Heat Radiation of NLVWSIE in A Semi-infinite Solid:

In this section we will study an Abel-type nonlinear Volterra integral equation

$y(x)=\frac{1}{\sqrt{\pi}} \int_{0}^{x} \frac{f(t)-y^{n}(t)}{\sqrt{x-t}} d t$,

Where $y(x)$ gives the temperature at the surface for all time. The physical problem which motivated consideration of (8) is that of determining (W.E.Olmstead, and R.A.Handelsman, 1976) the temperature in a semi-infinite solid, whose surface can dissipate heat by nonlinear radiation. At the surface, energy is supplied according to the given function $f(t)$, while radiated energy (Olmstead, Handelsman, 1976). escapes in proportion to $y^{n}(t)$. Equation $(8)$ may be rewritten as

$y(x)=\frac{1}{\sqrt{\pi}} \int_{0}^{x} \frac{f(t)}{\sqrt{x-t}} d t-\frac{1}{\sqrt{\pi}} \int_{0}^{x} \frac{1}{\sqrt{x-t}} y^{n}(t) d t$

As afirst application, we select $f(x)=\frac{1}{2}$ and $n=4$. Equation (9) becomes

$y(x)=\sqrt{\frac{x}{\pi}}-\frac{1}{\sqrt{\pi}} \int_{0}^{x} \frac{1}{\sqrt{x-t}} y^{4}(t) d t, \quad x \in[0,1]$

the expansion method using Chebyshev polynomials of thirsting of degree nas a basis function to handle (10). To determine an approximate solution of (10), the function $y(x)$ defined in $[0,1]$. This function maybe represented by the first kind shifted Chebyshev polynomials

$y(\bar{x}) \cong \sum_{i=0}^{\infty} T_{i}(\bar{x}) c_{i}$

If we truncated the series (11), then we can write (11) as follows:

$y(\bar{x}) \cong \sum_{i=0}^{N} T_{i}(\bar{x}) c_{i}=T(\bar{x}) C$

$y^{(4)}(\bar{x}) \cong\left(\sum_{i=0}^{N} T_{i}(\bar{x}) c_{i}\right)^{4}=(T(\bar{x}) C)^{4}$

where $T(\bar{x})=\left[T_{0}(\bar{x}), T_{1}(\bar{x}), T_{2}(\bar{x}), \ldots, T_{N}(\bar{x})\right], C=\left[c_{0}, c_{1}, c_{2}, \ldots, c_{N}\right]^{T}$

clearly $T$ is $1 \times(N+1)$ vectors and $C$ is $(N+1) \times 1$ vectors.

Then the aim is to find Chebyshev coefficients, that is the matrix $C$. We first substitute the Chebyshev nodes, which are defined by

$\bar{x}_{i}=\cos \left(\frac{(2 i+1) \pi}{2 N}\right), i=0,1,2, \ldots, N-1$ into (12) and (13)

then rearrange a new matrix form to determine $\mathrm{C}$ :

$y=f-k$

in which $k$ is the nonlinear integral part of (10) and

$y=\left(\begin{array}{c}y\left(\bar{x}_{0}\right) \\ y\left(\bar{x}_{1}\right) \\ \cdot \\ \cdot \\ \cdot \\ y\left(\bar{x}_{N}\right)\end{array}\right), f=\left(\begin{array}{c}f\left(\bar{x}_{0}\right) \\ f\left(\bar{x}_{1}\right) \\ \cdot \\ \cdot \\ \cdot \\ f\left(\bar{x}_{N}\right)\end{array}\right), k=\left(\begin{array}{c}k\left(\bar{x}_{0}\right) \\ k\left(\bar{x}_{1}\right) \\ \cdot \\ \cdot \\ \cdot \\ k\left(\bar{x}_{N}\right)\end{array}\right)$

by substituting (12) and(13) into (14) give nonlinear algebraic equations in $(N+1)$ unknown coefficients . These equations are solved byusing (Matlab R2017b) to obtain the unknown coefficients $C$ which are then substitute into (12) to get the approximate solution of (10).

\section{Example:}

In this section, we give application to the heat radiation of $\mathrm{NLVWSIE}_{\mathrm{s}}$, in the following ax ample, $N+1$ is the number of mesh points $y_{N}(\bar{x})$ denotes the approximate solution, where $N$ is the number of terms of the shifted Chebyshev polynomials, the computations have been performed by using Matlab R2017b.

We consider NL VWSIE shich arises in a heat radiation problem

$y(x)=\sqrt{\frac{x}{\pi}}-\frac{1}{\sqrt{\pi}} \int_{0}^{x} \frac{1}{\sqrt{x-t}} y^{4}(t) d t, \quad x \in[0,1]$

The approximate solution of heat radiation for $\mathrm{N}=2,4,6,8$ is given

When N=2 Roots of Shifted Chebyshev

$$
=\bar{x}_{i}
$$

$=\bar{x}_{i}$
0.066987298107780676618138414623532

0.5

0.93301270189221932338186158537647

Shifted Chebyshev coefficients is

$c_{0}=0.19431656, c_{1}=0.11457845, c_{2}=-0.02544027$

The approximate solution is 
$y(\bar{x})=-0.2035 \bar{x}^{2}+0.4327 \bar{x}+0.0543$

Elapsed time is 7.515077 seconds

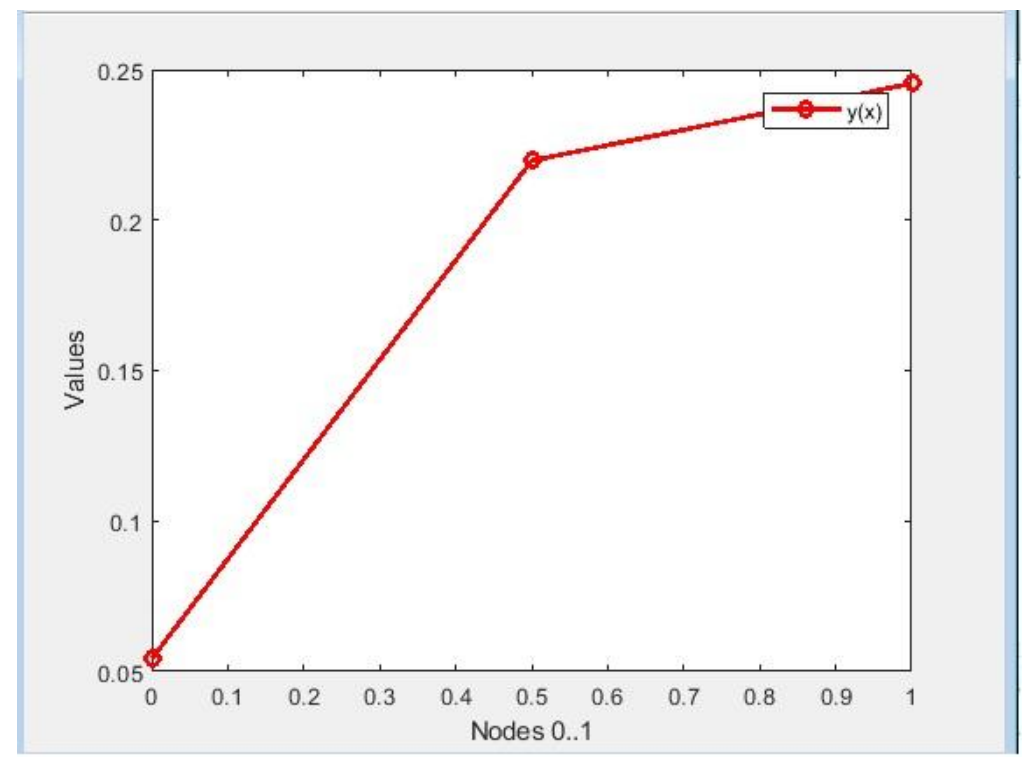

Fig. 3: The approximate solution of heat radiation of $\mathrm{NLVWSIE}_{\mathrm{s}}$ when $\mathrm{N}=2$.

When $N=4$ Roots of Shifted Chebyshev

$$
=\bar{x}_{i}
$$

0.024471741852423213941780333310309

0.20610737385376343541564702268046 0.5

0.79389262614623656458435297731954

0.97552825814757678605821966668969

Shifted Chebyshev coefficients is

$c_{0}=0.19279734, c_{1}=0.11797371, c_{2}=-0.03021305$

$c_{3}=0.00908978, c_{4}=-0.00329484$

The approximate solution is

$y(\bar{x})=-0.4217 \bar{x}^{4}+1.134 \bar{x}^{3}-1.205 \bar{x}^{2}+0.7467 \bar{x}+0.03223$

Elapsed time is 57.145148 secondes

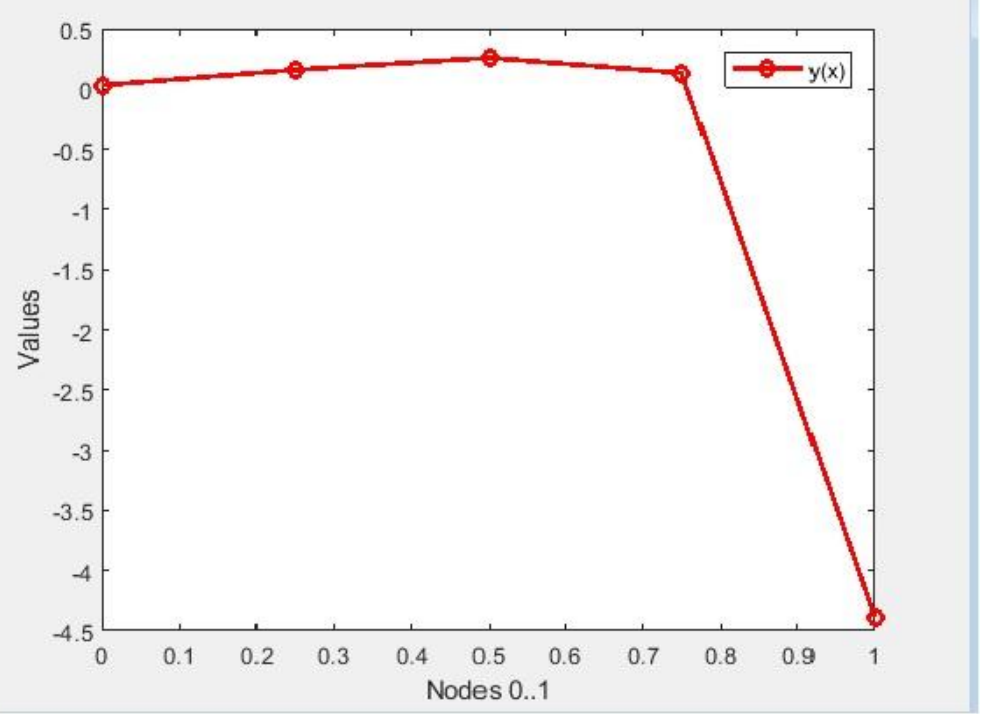

Fig. 4: The approximate solution of heat radiation of $\mathrm{NLVWSIE}_{\mathrm{s}}$ when $\mathrm{N}=4$.

When $\mathrm{N}=6$ Roots of shifted Chebyshev

$$
=\bar{x}_{i}
$$

0.012536043909088196490934158503034

0.10908425876598509564577773666297

0.28305813044122093976211583357582

0.5

0.71694186955877906023788416642418 
0.89091574123401490435422226333703

0.98746395609091180350906584149697

Shifted Chebyshev coefficients is

$c_{0}=0.19239228, c_{1}=0.11884472, c_{2}=-0.03122103, c_{3}=0.01037420, c_{4}=-0.00510760, c_{5}=0.00274968, c_{6}=-0.00121483$

The approximate solution is

$y(\bar{x})=-2.488 \bar{x}^{6}+8.872 \bar{x}^{5}-12.57 \bar{x}^{4}+9.073 \bar{x}^{3}-3.685 \bar{x}^{2}+1.063 \bar{x}+0.02288$

Elapsed time is 324.068763 secondes

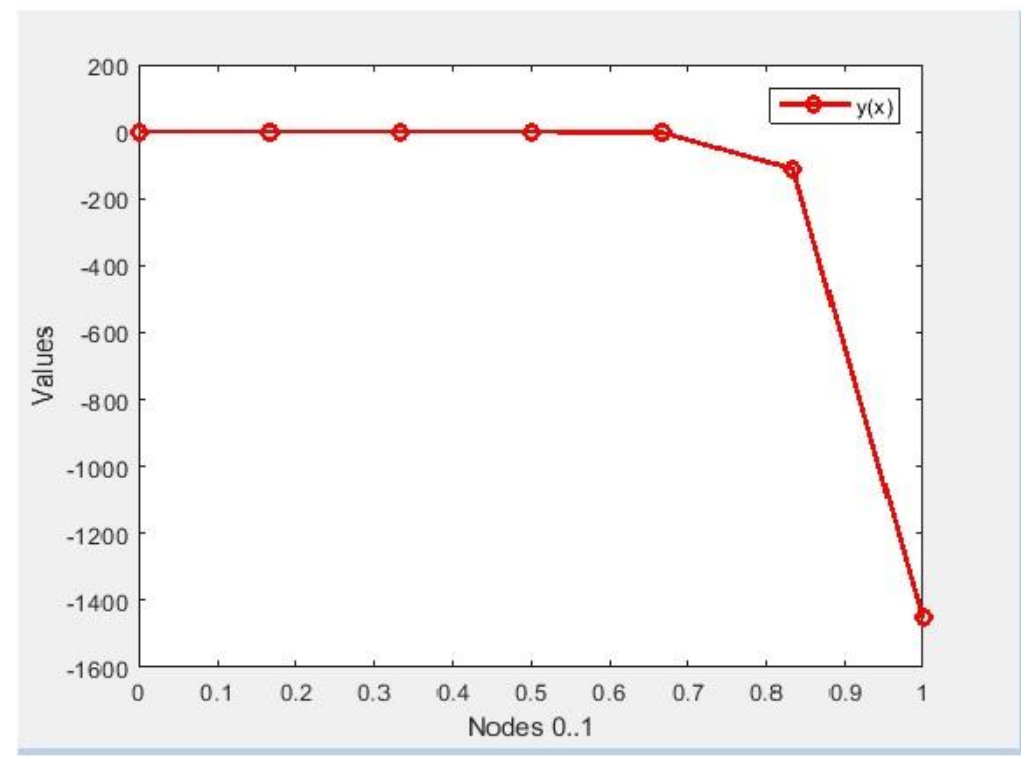

Fig. 5: The approximate solution of heat radiation of $\mathrm{NLVWSIE}_{\mathrm{s}}$ when $\mathrm{N}=6$.

When N=8 Roots of Shifted Chebyshev

$=\bar{x}_{i}$

0.0075961234938959703166284877052385

0.066987298107780676618138414623532

0.17860619515673033683867829504637

0.32898992833716563347795019265887

$2 / 1$

0.67101007166283436652204980734113

0.82139380484326966316132170495363

0.93301270189221932338186158537647

0.99240387650610402968337151229476

Shifted Chebyshev coefficients is

$c_{0}=0.19222276, c_{1}=0.11918909, c_{2}=-0.03159806, c_{3}=0.01080896, c_{4}=-0.00562415, c_{5}=0.00340694, c_{6}=-0.00208346, c_{7}=0.00120442, c_{8}$ $=-0.00055660$

The approximate solution is

$y(\bar{x})=-18.24 \bar{x}^{8}+82.82 \bar{x}^{7}-157.4 \bar{x}^{6}+162.3 \bar{x}^{5}-98.88 \bar{x}^{4}+36.37 \bar{x}^{3}-8.169 \bar{x}^{2}+1.375 \bar{x}+0.01775$

Elapsed time is 1646.952734 secondes 


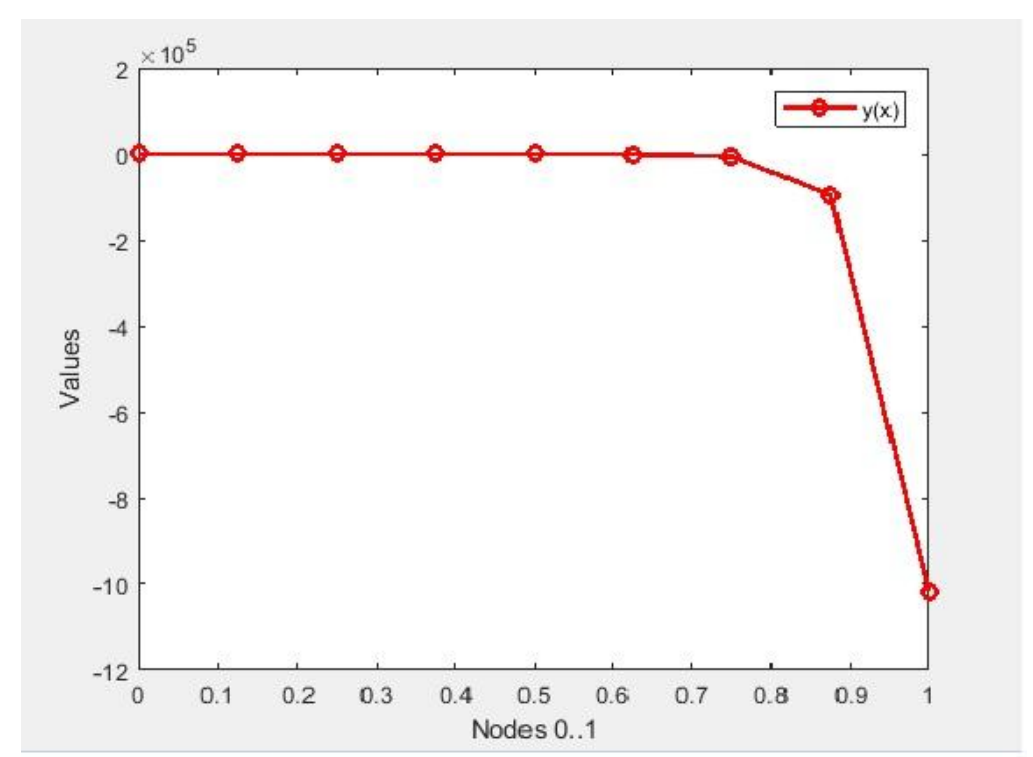

Fig. 6: The approximate solution of heat radiation of $\mathrm{NLVWSIE}_{\mathrm{s}}$ when $\mathrm{N}=8$.

\section{Conclusions:}

The expansion method using Shifted Chebyshev polynomials of the first kind of degree $\mathrm{n}$ is effectively to handle NLVWSIE's that describes of heat radiation. The expansion method using Shifted Chebyshev polynomials useful way to develop an analytic treatment for these kinds of nonlinear singular integral equations. Example we have reduced the solution of NLVWSIE's to the system of nonlinear equations by removing the singularity using an approximate point $\mathrm{t}$, and we have the following results: The proposed method is an accurate and efficient to solve NLVWSIEs.

\section{REFERENCES}

Abdul-Majid Wazwaz and Mona S. Mehanna, 2010. "The Combined Laplace - Adomain Method for Handling Singular Integral Equation of Heat Transfer", International Journal of Nonlinear Science., 10(2): 248-252.

Abdul-Majid, W., 2011. "Linear and Non- linear integral equations, Method and Applications”, Springer Heidelberg Dordrecht London New York.

Geankoplis, Christie John, 2003. Transport Processes and Separation Principles (4th ed.). Prentice Hall. ISBN 0-13-101367-X.

Mason, J.C., D.C. Handscomb, 2003. “Chebyshev Polynomials”, Boca Raton London New York Washington.

Tao, L. and H. Yong, 2006. "Extrapolation Method for Solving Weakly Singular Nonlinear Volterra Integral Equations of the Second kind", J. Math. Anal., 324: 225-237.

Rebelo, M. and T. Diogo, 2010. "A hybrid Collocation Method for a Nonlinear Volterra integral equation with weakly singular kernel”, Journal of Computational and Applied Mathematics, pp: 234: 1-10.

Franco, N.M.B., S. Mckee, 1985. "A family of high order product integration methods for an integral equation of Lighthill”, Int J. Comput. Math, 18: 17384.

Zeilon, N., 1924. "Sur Quelques Points De la Theorie De l' Equation Integrale d' Abel”, Arkiv. Mat .Astr. Fysik., 18: 1-19.

Gorenflo, R. and S. Vessella, 1991. "Abel Integral Equations: Analysis and Applications", Springer - Verlag, Berlin.

Gorenflo, R. and S. Vessella, 1991. “Abel Integral Equations”, Lecture Notes in Mathematics, Vol.1461, Springer, Berlin.

Olmstead, W.E. and R.A. Handelsman, 1976. Asymptotic solution to a class of nonlinear Volterra integral equations, II,SIAM J. 30(1): 180-189. 